\title{
Management of hyponatraemia and hypernatraemia during the Covid-19 pandemic: a consensus statement of the Spanish Society for Endocrinology (Acqua Neuroendocrinology Group)
}

\author{
Alberto Fernandez Martinez ${ }^{1}$ (D) - David Barajas Galindo ${ }^{2} \cdot$ Jorge Ruiz Sanchez $^{3}$ \\ Accepted: 7 January 2021 / Published online: 5 February 2021 \\ (C) The Author(s), under exclusive licence to Springer Science+Business Media, LLC part of Springer Nature 2021
}

\begin{abstract}
SARS-COV2 infection has swiftly become a pandemic disease of historic relevance and widely variable outcomes. This variable prognosis is related both to uneven damage, among others, to lungs, heart and kidneys, and to a multisystemic inflammatory reaction. All these factors are known to disrupt water balance and potentially induce hyponatraemia or hypernatraemia. Water balance disorders are known mortality and morbidity risk factors in several clinical scenarios and their proper management, though often complex and hazardous, can reduce mortality and length of hospitalization. Clinical uncertainty over COVID-19 outcome, the variety of organs involved in both the infection and water balance and difficulties in clinical examination due to risk of contagion might obstruct proper management of dysnatremic disorders. Thus, the Acqua Neuroendocrinology Group of the Spanish Society for Endocrinology (SEEN) has endeavoured to provide evidence and expert based recommendations on the management of hyponatraemia and hypernatraemia in COVID-19 patients.
\end{abstract}

Keywords COVID-19 · SARS-COV2 · Hyponatraemia · Hypernatraemia · Dysnatremia

On January 30th 2020, the World Health Organization officially declared SARS-COV2 infection(COVID-19) as a pandemic disease. As of today, over 84 million cases have been confirmed, including over 1.848 .000 deaths reported to WHO [1] . COVID-19 is a disease with widely variable outcomes, among which 19\% develop severe disease [2].Adverse clinical outcome correlates with multi-organ injury, maladaptive functions of the renin-angiotensin-aldosterone system and thirst and appetite abnormalities, all of which potentially disrupt water homeostasis [3]. A number of case reports describing hyponatraemia of various etiologies was initially published [4-7]. Later, a descriptive study of 16 patients with COVID-19-related hospitalization found $50 \%$ of them had hyponatraemia [8], whereas the HOPE study, an international registry of 4664 hospitalized COVID-19 patients, found that

Alberto Fernandez Martinez

afernamed@hotmail.com

1 Endocrinology Department, Hospital Universitario de Mostoles, Madrid, Spain

2 Endocrinology Department, Hospital Universitario de Leon, Leon, Spain

3 Hospital Clínico Universitario San Carlos, Madrid, Spain
$20.5 \%$ and $3.7 \%$ had hyponatraemia and hypernatraemia, respectively [9]: both hyponatraemia and hypernatraemia were generally mild (130-134 mEq/1 in $16.7 \%)$, severe hyponatraemia $(<120 \mathrm{mEq} / \mathrm{l})$ was found only in $0.4 \%$. Multivariable logistic regression analysis showed that male sex and age $>70$ years were independently associated with hyponatraemia at admission, whereas smoking, tachypnea and serum creatinine $>1.5 \mathrm{mg} / \mathrm{dl}$ bore a positive, independent association with admission hypernatraemia. Finally, the HOPE registry revealed that hyponatraemia was an independent risk factor for mortality (OR 1.5, 95\% CI 1.08-2.09; $p=$ 0.016 ) and sepsis (OR 1.87, 95\% CI 1.23-2.66; $p<0.001$ ), as was the case also for hypernatraemia (Mortality OR 2.38, 95\% CI 1.18-4.78; $p=0.015$. Sepsis OR 3.78, 95\% CI. 1.98-7.22; $\mathrm{p}<0.001)$ [9].

Patients with chronic disorders of water homeostasis, such as diabetes insipidus (DI) or chronic hyponatraemia $(\mathrm{CH})$ are prone to suffering exacerbations of water imbalance during COVID-19 pandemic, either due to the loss of follow-up in non-infected patients with DI or chronic hyponatraemia or the impact of SARS-COV2 infection on water homeostasis. Thus, the European Society of Endocrinology published expertbased recommendations on the management of diabetes insipidus and hyponatraemia in the time of COVID-19 [10]. However, published literature on COVID-19 and its related 
complications increases swiftly; meanwhile, further waves and incidence peaks are expected in the near future, and these recommendations were based on preliminary data on the disease and require further coverage on practical aspects of fluid and electrolyte balance in these patients.

According to published data and clinical expertise, patients with DI or $\mathrm{CH}$ present additional challenges upon COVID-19 infection:

- COVID-19 induced hyponatraemia or hypernatraemia, present in $20.5 \%$ and $3.7 \%$ respectively of hospitalized COVID-19 patients without underlying water balance disorders [9]

- Challenges in the management of DI or $\mathrm{CH}$ during COVID-19 infection

- Limitations for the clinical evaluation due to risk of contagion

- Pharmacological interactions between sodium directed therapies (i.e. tolvaptan, desmopressin) and COVID-19 treatments (i.e. tocilizumab)

Management of patients with chronic water balance disorders during COVID-19 pandemic requires careful consideration of two opposing paradigms:

1) Careful follow-up and treatment of DI and $\mathrm{CH}$ patients, who might worsen their water balance homeostasis if SARS-COV2 infection occurs

2) Reducing clinic attendance and non-urgent tests where possible to avert patient's risk of infection

Thus, the Neuroendocrinology Group of the Spanish Society for Endocrinology (SEEN) has endeavoured to provide recommendations both for the chronic management of DI and $\mathrm{CH}$ during this pandemic and for the evaluation of newonset water balance disorders in COVID-19 patients. Where possible we have attempted to develop evidence-based guidelines, whereas expert-based consensus was provided when evidence was lacking.

\section{Specific risks in patients with chronic hyponatraemia or diabetes insipidus and COVID-19}

Mismanagement of water balance disorders during acute illness can worsen morbidity and mortality $[11,12]$. Thus, adequate evidence-based treatment of $\mathrm{CH}$ or DI, as previously described in European and American Hyponatraemia Guidelines $[13,14]$ and in the European Society for Endocrinology statement on the management of these disorders during COVID-19 pandemic [10], may improve outcomes, including risk of hospitalization and therefore SARS-COV2 exposure.

COVID-19, particularly in severe forms, can induce numerous electrolyte abnormalities that might be particularly severe in patients with chronic water balance abnormalities [2]. According to published literature, these are some of the most common alterations that might complicate the management of patients with wáter balance abnormalities and SARSCOV2 infection:

a. Complications linked to the COVID-19 pandemic that might worsen the outcome of patients with either DI or $\underline{\mathrm{CH}}$ :

- COVID-19-related electrolyte abnormalities: hyponatraemia, hypernatraemia, hypokalemia, mixed alcalosis (respiratory alcalosis due to hyperventilation, metabolic alcalosis linked to hypovolaemia)

- Loss of follow-up of CH or DI due to patient's and/or health care worker fear of contagion or work overload by the health care worker during COVID-19 outbreaks.

- Difficulties accessing water due to psychosocial factors, such as social isolation during confinement and anxiety or depression linked to outbreaks, can be particularly harmful in $\mathrm{CH}$ and DI patients. Futhermore, water intake can activate mesolimbic reward circuits in the central nervous system, which could lead to psychogenic polydipsia in these patients and ultimately worsen water balance [15].

b. DI-specific risks during COVID-19 pandemic:

- Loss of follow-up during the pandemic might lead to undetected hyponatraemia due to desmopressin overdose, particularly when administered through intranasal route [16]. SARS-COV2 infection might also increase antidiuresis. However, larger series with more in-depth description of volume status and serum and urine electrolytes are required.

- During SARS-COV2 infection, extrarenal water loses might increase due to hyperpyrexia, hyperventilation and/or diarrhoea and cause hypernatraemia. This could worsen further if either the patient or health care workers do not ensure timely administration of desmopressin and monitorization of sodium levels take place regularly in patients with DI [17].

- Patients with central DI often suffer as well from adrenal insufficiency and hypothyroidism. Timely administration of thyroid hormones and glucocorticoid replacement is paramount, increasing the dose of glucocorticoids during intercurrent illnesses as described in clinical guidelines [18].

- Obesity is common in patients with central DI, particularly when other pituitary hormonal deficiencies or 
hypersecretion syndromes (i.e. Cushing's disease) are present. Obese patients suffer from worse COVID-19 related prognosis [19], which highlights the need to reduce contagion risk in these patients.

- Metabolic comorbidities in patients with central DI that could worsen COVID-19 prognosis: diabetes mellitus, hypertension, obstructive sleep apnea

- Increased risk of thrombosis in DI due to hypernatraemia, which might worsen during SARS-COV2 infection, also associated with hypercoagulability, or in non-infected patients due to confinement, increased weight and lack of physical activity [20, 21]. Therefore, thromboprophylaxis should be systematically considered in COVID-19 patients with chronic DI.

c. Chronic hyponatraemia specific risks during COVID-19 pandemic:

a. Patients with chronic SIADH might suffer from decompensated hyponatraemia during COVID-19 infection due to thirst abnormalities during acute illness, lack of treatment adherence (i.e. tolvaptan, urea...) and/or treatment monitorization.

b. Changes in volume status during intercurrent illness, i.e. hypovolaemia caused by vomiting or hyperpirexia with increased perspiration. Both tolvaptan and urea are licensed for use during euvolemic hyponatraemia due to SIADH, but patients with hyponatraemia might change volume status during acute illness.

iii. Chronic comorbidities common in patients with SIADH that might worsen COVID-19 prognosis: cancer, respiratory disorders, central nervous system disorders...

\section{Management of patients with DI during COVID-19 pandemic}

a. Non-infected patients:

- Outpatient follow-up according to clinical guidelines [11]. If patients have preserved thirst sensation and are capable of regulating water intake and maintain treatment adherence, follow up could be performed through telemedicine (phone contact, videocalls...) with lab tests as required.

- More stringent follow-up is indicated in the following scenarios: adipsic DI, neurocognitive impairment that concerns water intake or previous episodes of hyponatraemia due to desmopressin overdose.

b. COVID-19 in patients with chronic DI:

Cooperation between health care workers dealing with COVID-19 and clinicians with expertise in water balance disorders is paramount in this scenario. Some aspects of DI treatment may vary according to COVID-19 severity [2, 22] and water balance status on presentation.

\section{Mild or moderate COVID-19}

- Outpatients with chronic DI and mild to moderate COVID-19 should at least have one blood and urine test measuring osmolality, sodium, creatinine and potassium during the acute phase and whenever the medical team has identified significant changes in factors influencing water balance (fever, loss of thirst sensation, hyperventilation...)

- Hospital admission of COVID-19 patients with chronic DI, besides following COVID-19 evolution, should be implemented if there are significant changes in plasma sodium levels, loss of thirst or new-onset neurocognitive impairment that hinders outpatient management (see section on "severe COVID-19")

- Inpatient management: if admission is required for the treatment of COVID-19, conscious patients with intact thirst may maintain their usual desmopressin replacement regime with daily monitoring of plasma and urine sodium, potassium, creatinine and osmolality during the acute phase of COVID-19 as inpatients. Samples should be extracted one hour before the morning doseof desmopressin. It is strongly recommended that subjects with chronic DI wear medical alert bracelets or cards and duly inform their medical team of their condition at first contact. An example of medical alert card for diabetes insipidus created by our group is provided in Fig. 1.

\section{Severe COVID-19 in patients with chronic DI}

- It is mandatory to check daily fluid balance: oral and parenteral fluid administration (including fluids needed to administer parenteral drugs), diuresis and extrarenal fluid losses (i.e. diarrhea, vomiting, fever...).

- Daily monitoring of plasma and urine sodium, potasium, creatinine and osmolality

- Parenteral desmopressin administration should be first choice in severe COVID-19 patients to ensure stable and predictable desmopressin activity; conversion to parenteral doses should consider the pharmacokinetic bioequivalence among different administration routes: 1 mcg of parenteral desmopressin equals $10 \mathrm{mcg}$ of intranasal and $100 \mathrm{mcg}$ of oral desmopressin; thus, a patient 


\section{IMPORTANT}

\section{DIABETES INSIPIDUS}
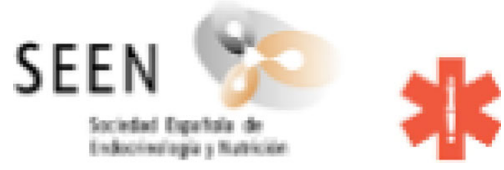

THIS PATIENT NEEDS DAILY

REPLACEMENT THERAPY

WITH DESMOPRESSIN.

If severely unwell, vomiting or diarrhoea, give desmopressin at the usual dose and check liquid balance

Name

Social security number / Contact

phone number and person

Fig. 1 Medical alert card for patients with diabetes insipidus

on chronic treatment with intranasal desmopressin 10 mcg bd should be initially changed to $1 \mathrm{mcg}$ parenteral DDAVP bd. It is advisable to ensure osmotic homeostasis is maintained through at least daily monitoring of plasma and urine osmolality [17]

- If intercurrent illness or underlying chronic conditions impair the patient's ability to cover daily water, electrolytes and/or nutrients needs through oral intake, osmotic balance and nutritional status should be maintained using parenteral route. Standard fluid requirements include $25-30 \mathrm{ml} / \mathrm{kg}$-day of water, $1 \mathrm{mmol} / \mathrm{kg}$-day of potassium, sodium and chloride and $100-150 \mathrm{~g} /$ day of glucose. If extrarenal water loss increases, corrections should be implemented accordingly (diarrhea, vomiting...); i.e. daily water administration should increase by $100 \mathrm{ml}$ for each temperature degree above $37{ }^{\circ} \mathrm{C}$ )

- If the patient needs enteral or parenteral nutritional support according to stablished guidelines, water, electrolyte and glucose replacement should be included in nutritional formulations [23].

- In obese patients with normal nutritional status, water, glucose and electrolyte requirements will be tailored to the patient's ideal weight. Obesity often does not preclude malnutrition, which should be equally screened and treated.

- If a patient with chronic DI develops hyponatraemia during COVID-19, three underlying factors should be identified and treatment:

- Desmopressin overdose, that can be corrected reducing the dose of DDAVP and monitoring clinical evolution (consciousness, gait, speech...) and biochemical evolution

- Secondary adrenal insufficiency, common in patients with central DI and whose under-replacement might lead to hyponatraemia. To our knowledge, no case of adrenal insufficiency due to SARS-COV2 infection has been published so far.

- Patients with hypopituitarism often suffer from metabolic comorbidities that make them prone to developing stressinduced hyperglycemia during COVID-19. Hyperglycemia might provoke increased osmotic diuresis and disturb water balance, particularly in patients with chronic DI. This situation should be dealt with treating hyperglycemia according to evidence-based guidelines and covering fluid losses orally or parenterally. Desmopressin dose, however, should not be modified if osmotic diuresis is the only factor altering water balance unless hypernatraemia develops.

\section{Management of patients with chronic SIADH in COVID-19}

a. Non-infected patients:

- Patients with chronic SIADH should regularly receive clinical examination that includes the assessment of internal jugular vein pressure. These data are needed to appraise dose changes and even interruption of hyponatraemia pharmacotherapy (tolvaptan, urea...). Thus, patients with pharmacologically treated and chronic hyponatraemia are not fit for telemedicine follow-up appointments wherever this is problem is concerned.

- Patients with chronic SIADH usually suffer from malnutrition as well due to common precipitating factors (multimorbidity). Malnutrition is an absolute contraindication for water restriction as a treatment for hyponatraemia, as water-restricted dieting might worsen nutritional outcomes. In patients with SIADH and without malnutrition, confinement and/or loss of social interaction might also hinder adherence to water restriction; in this situation pharmacotherapy for hyponatraemia should also be considered according to stablished guidelines $[9,10]$. 
- New-onset hyponatraemia and COVID-19:

- Hyponatraemia, whatever the underlying causes, represents a risk factor for mortality and increased length of hospitalization. Therefore, hyponatraemia should be identified and treated accordingly in COVID-19 patients [12]

- Diagnosis of new-onset hyponatraemia in COVID-19 patients should initially include plasma and urine osmolality, sodium and potassium and clinical assessment of volume status. Further etiological tests should be guided by the patient's underlying conditions and risk factors, volume status and evolution. COVID-19 might be associated with multiple underlying disorders that might precipitate or aggravate hyponatraemia (Fig. 2). Among those etiologies, endocrinological assessment should include free thyroxine and thyroid stimulating hormone measurement, as COVID-19 can be associated with thyroid dysfunction [24], as well as diagnostic tests for adrenal insufficiency (9 am cortisol and/or synacthen test). Adrenal failure might be elicited by direct SARS-COV2 infiltration of adrenal cells or by dexamethasone-induced adrenal suprression [18, 25].

- Patients suffering from COVID-19 should not be waterrestricted in order to treat hyponatraemia. SARS-COV2 infection is often associated with malnutrition, increased perspiration and hyperventilation, and these factors may aggravate the adverse effects of water restriction. [2, 3, 23].

- Monitoring of COVID-19 patients with hyponatraemia should include daily measurements of plasma and urine sodium, potassium, creatinine and osmolality and clinical assessment of volume status (measurement of internal jugular vein pressure, intraocular pressure...). More intensive monitoring could be required in patients with severe hyponatraemia or those with rapidly evolving hyponatraemia.

- Hyponatraemia pharmacotherapy during COVID-19:

i. Indications for hyponatraemia pharmacotherapy in COVID-19 patients: euvolemic hyponatraemia with plasma sodium $>120 \mathrm{mEq} / 1$, after excluding adrenal insufficiency and hypothyroidism. Most COVID-19 patients will not be suitable candidates for water restriction as a treatment for hyponatraemia due to malnutrition, loss of thirst and increased extraurinary water loss. These patients should therefore be treated with tolvaptan or urea according to clinical guidelines [13, 14].

ii. Treatment interactions between hyponatraemia and SARS-COV2 pharmacotherapy: described in Table 1 and for each drug in its specific section.

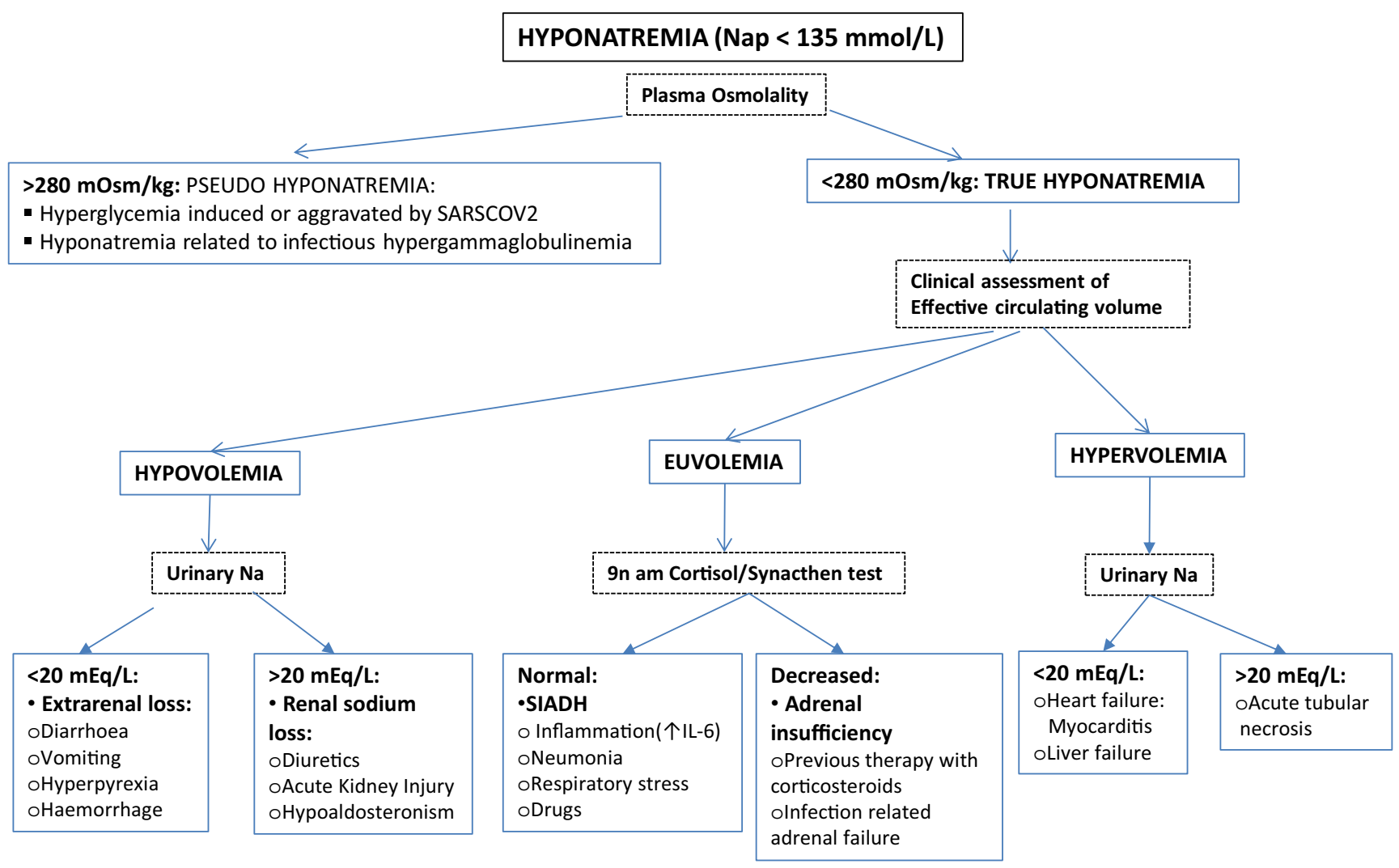

Fig. 2 Hyponatraemia diagnostic algorithm in COVID-19 patients 
Table 1 Pharmacological interactions between COVID-19 and hyponatraemia pharmacotherapy [28, 29, 33]

\begin{tabular}{|c|c|c|c|c|c|}
\hline $\begin{array}{l}\text { Hyponatraemia } \\
\text { drug }\end{array}$ & Lopinavir/ritonavir & Tocilizumab & Remdesivir & Hydroxychloroquine & Dexamethasone \\
\hline Tolvaptan & $\begin{array}{l}\uparrow \text { Tolvaptan } \\
\text { concentrations } \\
\text { (CYP3A4 } \\
\text { inhibition) }\end{array}$ & $\begin{array}{l}\downarrow \text { Tolvaptan concentrations } \\
\text { (CYP3A4 activation) }\end{array}$ & $\begin{array}{l}\uparrow \text { Tolvaptan } \\
\text { concentrations } \\
\text { (CYP3A4 } \\
\text { inhibition) }\end{array}$ & None & None \\
\hline Urea & \multicolumn{5}{|c|}{ No clinical experience published in COVID-19 } \\
\hline Furosemide & - & - & - & - & $\begin{array}{c}\text { Corticosteroids may } \\
\uparrow \text { hypokalemia }\end{array}$ \\
\hline
\end{tabular}

- Patients with plasma sodium $<120 \mathrm{mEq} / 1$ regardless of their clinical status or $>120 \mathrm{mEq} / 1$ with severe neurological symptoms should be treated with hypertonic saline serum according to published evidence-based guidelines $[13,14]$

- Euvolemic or hypervolemic hyponatraemia, particularly with urine osmolality $>350 \mathrm{mOsm} / \mathrm{kg}$, may be treated with loop diuretics after an oral load of 4-5 $\mathrm{g}$ of $\mathrm{NaCl}$ [26]

- Hypovolaemic hyponatraemia may be treated with isotonic saline fluids, no more than $30 \mathrm{ml} / \mathrm{kg}$-day to avoid hypercorrection [27].

\section{c.1 Tolvaptan in COVID-19 patients:}

- Initialdose: $7.5 \mathrm{mg} /$ day

- Contraindicated if severe liver or kidney failure (Child C orGFR $<30 \mathrm{ml} / \mathrm{min}$ ).

- Precautions:

- Treatment with lopinavir/ritonavir inhibits CYP3A4, a potent pharmacological interaction with tolvaptan that increases plasma levels of the latter and could increase its acuaretic effect. Simultaneous use of both compounds should be closely monitored [28]

- Tocilizumab enhances CYP3A4 activity, which could decrease tolvaptan's circulating levels and its acuaretic effect [29]

- No significant interactions have been described between hydroxychloroquine and any drug licensed for use in hyponatraemia

\section{c.2 Urea in COVID-19:}

- No clinical experience has been published with urea in SARS-COV2 infected patients with hyponatraemia

- Warnings:

- Urea may decrease thirst, treated patients should be monitored, measuring intake and loss of fluids, changes in thirst, urine volume and new-onset nocturia.

\section{c. 3 Furosemide in COVID-19 patients:}

- Patients with euvolemic or hypervolemic hyponatraemia with urine osmolality $>350 \mathrm{mOsm} / \mathrm{kg}$.

- Furosemide may exacerbate hypokalemia in COVID-19 patients, who also display high activity of the reninangiotensin-aldosterone axis [3, 30].

\section{Management of hypernatraemia in COVID-19}

Hypernatraemia is a common laboratory abnormality in COVID-19 patients, found in 3,7\% of patients hospitalised due to COVID-19 in the HOPE registry [9]. Similarly to hyponatraemia, increased plasma sodium levels are an adverse prognostic factor in non-COVID patients although this awaits confirmation in COVID-19 series. Hypernatraemia in COVID-19 develops due to an inbalance between increased water loss (hyperpyrexia, diarrhoea, vomiting, polyuria due to hyperglycemia or acute kidney injury...) and decreased water supply (anorexia with adipsia, altered consciousness, insufficient parenteral replacement...) [31,32].

Diabetes insipidus as a direct consequence of SARS-COV2 has not been pathophysiologically described so far. Therefore, while awaiting descriptive studies of hypernatraemia in this population, it could be safely assumed that COVID-19 patients with hypernatraemia mostly suffer increased extrarenal water loss.

Treatment of hypernatraemia in COVID-19 patients is similar to that described in the general population, based on parenteral fluid replacement [31].

General recommendations:

- Thrombosis prevention: hypernatraemia and SARSCOV2 infection are both independent thrombosis risk factors. If no contraindication is found, low weight heparine profilaxis should be initiated on admission.

- Plasma and urine sodium measurement every 12-24 h 
- Objective: reduction of sodium levels $<10 \mathrm{mEq} / 1$ every $24 \mathrm{~h}$ to reduce the risk of cerebral edema

- Severe hypernatraemia with loss of consciousness

- Dextrose $5 \%$ serum at $1.35 \mathrm{ml} / \mathrm{kg}$-hour

- If there is clinical hypovolaemia, administer isotonic serum (saline serum $0,9 \%$ or Ringer) parallel to dextrose at $30 \mathrm{ml} / \mathrm{kg} /$ day to increase effective circulating volume. Patients with increased risk of cerebral edema (liver failure, heart failure) may receive instead saline serum $0,45 \%$ at $30 \mathrm{ml} / \mathrm{kg}$-day.

Data availability not applicable.

\section{Compliance with ethical standards}

Conflict of interest none relevant to the article.

Ethics approval not applicable.

\section{References}

1. WHO Coronavirus Disease dashboard [Internet]. Available from: https://covid19. who.int. Accessed 3 Oct 2020.

2. Berlin DA, Gulick RM, Martinez FJ. Severe Covid-19. N Engl J Med. 2020.

3. Gupta A, Madhavan MV, Sehgal K, Nair N, Mahajan S, Sehrawat TS, et al. Extrapulmonary manifestations of COVID-19. Nat Med. $2020 \mathrm{Jul} ; 26(7): 1017-32$.

4. De La Flor Merino JC, Mola Reyes L, Linares Gravalos T, Roel Conde A, Rodeles Del Pozo M. An unusual case of severe acute hyponatraemia in patient with COVID-19 infection. Nefrol Publicacion Of Soc Espanola Nefrol. 2020 Jun;40(3):356-8.

5. Mansoor E, Khoudari G, Abou Saleh M, Elfadawy N, Cooper GS, Katz J, et al. The many faces of COVID-19: atypical presentation of COVID-19 in a patient with Crohn's disease with acute diarrhea leading to severe hypovolemic Hyponatraemia-a case report. Am J Gastroenterol. 2020 Aug; 19.

6. Tee LY, Yap B, Sidhu GK, Goh KS, Rosario BH. Atypical presentation of COVID-19 in an older adult: lethargy and vomiting from severe hypovolemic Hyponatraemia. Geriatr Gerontol Int. 2020 $\mathrm{Jul} ; 8$.

7. Khan AA, Ata F, Munir W, Yousaf Z. Fluid replacement versus fluid restriction in COVID-19 associated Hyponatraemia. Cureus. 2020 Jul 8;12(7):e9059.

8. Aggarwal S, Garcia-Telles N, Aggarwal G, Lavie C, Lippi G, Henry BM. Clinical features, laboratory characteristics, and outcomes of patients hospitalized with coronavirus disease 2019 (COVID-19): early report from the United States. Diagn Berl Ger. 2020;7(2):91-6.

9. Ruiz-Sánchez JG, Núñez-Gil IJ, Cuesta M, Rubio MA, MarounEid C, Arroyo-Espliguero R, et al. Prognostic Impact of Hyponatraemia and Hypernatraemia in COVID-19 Pneumonia. A HOPE-COVID-19 (Health Outcome Predictive Evaluation for COVID-19) Registry Analysis. Front Endocrinol [Internet]. 2020 [cited 2021 Jan 6]; 11. Available from: https://doi.org/10.3389/ fendo.2020.599255/full.

10. Christ-Crain M, Hoorn EJ, Sherlock M, Thompson CJ, Wass JAH. ENDOCRINOLOGY IN THE TIME OF COVID-19: management of diabetes insipidus and hyponatraemia. Eur J Endocrinol. 2020 Jul;183(1):G9-15.

11. Baldeweg, S.E., Ball, S B, Brooke, A, Gleeson, H.K.G., Levy, M. J., Prentice, M., et al. Society for Endocrinology Clinical Guidance: Inpatient Management of Cranial Diabetes Insipidus. Endocrine connections. 2018.

12. Cuesta M, Garrahy A, Slattery D, Gupta S, Hannon AM, McGurren $\mathrm{K}$. Mortality rates are lower in SIAD, than in hypervolaemic or hypovolaemic hyponatraemia: Results of a prospective observational study. Clin Endocrinol. et al., 2017.

13. Verbalis J. Goldsmith, Gd, Greenberg, mark, Schrier, sterns, Rh S. Hyponatraemia treatment guidelines 2007: expert panel recommendations. Am J Med. 2007 Nov;126(10 Suppl 1):S1-42.

14. Spasovski G, Vanholder R, Allolio B, Djillali A, Ball S, Bichet D, et al. Clinical practice guideline on diagnosis and treatment of Hyponatraemia. Eur J Endocrinol. 2014;170(3):G1-47.

15. Hew-Butler T, Smith-Hale V, Pollard-McGrandy A, VanSumeren M. Of mice and men-the physiology, psychology, and pathology of Overhydration. Nutrients. 2019 Jul;11(7):1539.

16. Kataok Y, Nishida S, Hirakawa A, Oiso Y, Arima H. Comparison of incidence of hyponatraemia between intranasal and oral desmopressin in patients with central diabetes insipidus. Endocr J. 2015;62(2):195-200.

17. Lamas C, del Pozo C, Villabona C. Clinical guidelines for management of diabetes insipidus and syndrome of inappropriate antidiuretic hormone secretion after pituitary surgery. Endocrinol Nutr Organo Soc Espanola Endocrinol Nutr. 2014;61(4):15-24.

18. Arlt W, Baldeweg SE, Pearce SHS, Simpson HL. ENDOCRINOLOGY IN THE TIME OF COVID-19: management of adrenal insufficiency. Eur J Endocrinol. 2020 Jul;183(1): G25-32.

19. Caci G, Albini A, Malerba M, Noonan DM, Pochetti P, Polosa R. COVID-19 and Obesity: Dangerous Liaisons. J Clin Med. 2020;9(8).

20. Connors JM, Levy JH. COVID-19 and its implications for thrombosis and anticoagulation. Blood. 2020;135(23):2033-40.

21. Miljic D, Miljic P, Doknic M, Pekic S, Stojanovic M, Petakov M, et al. Adipsic diabetes insipidus and venous thromboembolism (VTE): recommendations for addressing its hypercoagulability. Horm Athens Greece. 2014 Sep;13(3):420-3.

22. Gandhi RT, Lynch JB, del Rio C. Mild or Moderate Covid-19. N Engl J Med. 2020.

23. Arkin N, Krishnan K, Chang M, Bittner E. Nutrition in critically ill patients with COVID-19: challenges and special considerations. Clin Nutr Edinb Scotl. 2020 Jul;39(7):2327-8.

24. Scappaticcio L, Pitoia F, Esposito K, Piccardo A, Trimboli P. Impact of COVID-19 on the thyroid gland: an update. Rev Endocr Metab Disord. 2020;25:1-13.

25. Ferraù F, Ceccato F, Cannavò S, Scaroni C. What we have to know about corticosteroids use during Sars-Cov-2 infection. J Endocrinol Investig. 2020;28:1-9.

26. Ortola A, Crespo I, Ruiz-Gracia T, Gomez-Hoyos E, Cuesta M, Penso R, et al. The use of an oral salt load followed by furosemide in the treatment of euvolemic SIADH-induced hyponatraemia. In BioScientifica; 2015 [cited 2020 Aug 30]. Available from: https:// www.endocrine-abstracts.org/ea/0037/ea0037ep672

27. Ruiz-Sánchez JG, Meneses D, Álvarez-Escolá C, Cuesta M, CallePascual AL, Runkle I. The Effect of the Dose of Isotonic Saline on the Correction of Serum Sodium in the Treatment of Hypovolemic Hyponatremia. Journal of Clinical Medicine. 2020;9(11): 3567.https://www.researchgate.net/publication/329923614_ Hyponatraemia_hipovolemica_dosis_umbral_de_suero_salino_ fisiologico_para_evitar_sobrecorreccion

28. Shoaf SE, Bricmont P, Mallikaarjun S. Effects of CYP3A4 inhibition and induction on the pharmacokinetics and pharmacodynamics 
of tolvaptan, a non-peptide AVP antagonist in healthy subjects. Br J Clin Pharmacol. 2012;73(4):579-87.

29. Schmitt C, Kuhn B, Zhang X, Kivitz AJ, Grange S. Disease-drugdrug interaction involving tocilizumab and simvastatin in patients with rheumatoid arthritis. Clin Pharmacol Ther. 2011;89(5):73540.

30. Tomasoni D, Italia L, Adamo M, Inciardi RM, Lombardi CM, Solomon SD, et al. COVID 19 and heart failure: from infection to inflammation and angiotensin II stimulation. Searching for evidence from a new disease. Eur J Heart Fail. 2020;15.

31. Muhsin SA, Mount DB. Diagnosis and treatment of hypernatraemia. Best Pract Res Clin Endocrinol Metab. 2016;30(2):189-203.
32. Rondon-Berrios H, Argyropoulos C, Ing TS, Raj DS, Malhotra D, Agaba EI, et al. Hypertonicity: clinical entities, manifestations and treatment. World J Nephrol. 2017 Jan 6;6(1):1-13.

33. Widmer P, Maibach R, Künzi UP, Capaul R, Mueller U, Galeazzi $\mathrm{R}$, et al. Diuretic-related hypokalaemia: the role of diuretics, potassium supplements, glucocorticoids and beta 2-adrenoceptor agonists. Results from the comprehensive hospital drug monitoring programme, berne (CHDM). Eur J Clin Pharmacol. 1995;49(1-2): $31-6$.

Publisher's note Springer Nature remains neutral with regard to jurisdictional claims in published maps and institutional affiliations. 